\title{
Research Paper: Stress-Induced Spatial Memory Deficit Reversed by Basolateral Amygdala NMDA Receptor Inhibition in Male Wistar Rats
}

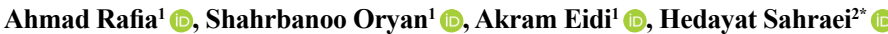 \\ 1. Department of Biology, Faculty of Basic Sciences, Science and Research Branch, Tehran, Iran.
}

2. Neuroscience Research Center, Baqiyatallah University of Medical Sciences, Tehran, Iran.

\begin{tabular}{|c|c|}
\hline \multirow{3}{*}{ 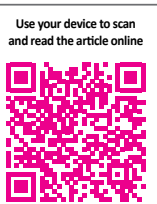 } & \\
\hline & $\begin{array}{l}\text { Aftation: Rafia, A., Oryan, Sh., Eidi, A., \& Sahraei, H. Stress-Induced Spatial Memory Deficit Reversed by Basolateral } \\
\text { Amygdala NMDA Receptor Inhibition in Male Wistar Rats. Basic and Clinical Neuroscience, 11(4), 447-456. http://dx.doi. } \\
\text { org/10.32598/bcn.11.4.15.11 }\end{array}$ \\
\hline & doij http://dx.doi.org/10.32598/ben.11.4.15.11 \\
\hline
\end{tabular}

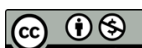

Article info:

Received: 11 May 2019

First Revision: 24 Jun 2019

Accepted: 10 Feb 2020

Available Online: 01 Jul 2020

Keywords:

Basolateral amygdala, Glutamate NMDA receptors, Memantine, Spatial learning and memory, Stress

\begin{abstract}
A B S T RA C T
Introduction: The present study investigated the role of the Basolateral Amygdala (BLA) N-methyl-D-aspartate (NMDA) receptors in stress-induced spatial memory disturbance among the male Wistar rats.

Methods: The male Wistar rats (Average weight $=200 \mathrm{~g}$ ) were cannulated bilaterally in the BLA, and entered the study $(n=6-8)$ after one week. They received seven electro-foot-shock stress sessions on seven consecutive days. Memantine $(0.1,1$, and $5 \mu \mathrm{g} / \mathrm{rat})$ or saline $(0.5 \mu \mathrm{L} /$ rat) was injected into the BLA, five minutes before each stress session. The control groups received the same doses of memantine and no stress. After the end of the stress sessions, blood samples were taken from all animals to evaluate their plasma corticosterone. Also, the spatial learning and memory of the study animals were evaluated using the Barnes maze method. The animals experienced five consecutive days of training on the maze for spatial learning. On the sixth day, their spatial memory was evaluated on the maze. Time, distance, the number of errors, and the taking strategy for reaching the target hole were considered as the parameters for the spatial learning and memory evaluation.

Results: Stress increases the plasma corticosterone level, while memantine preadministration reduces the stress effects. Besides, stress increases the time and distance to the target hole and the number of errors. Stress changed the animals' strategy from serial to random type. However, the intra-BLA memantine reversed all the disturbances induced by the stress.

Conclusion: This study indicated that the BLA glutamate NMDA receptors modulate the effect of stress on spatial learning and memory deficit.
\end{abstract}




\section{Highlights}

- Chronic electro foot shock stress can impaired the spatial learning and memory as revealed by Barnes maze paradigm.

- The impairments was altered by memantine pre microinjection into the BLA.

- Stress changes the strategy to achieving the target hole and memantine also inhibited this issue.

\section{Plain Language Summary}

The bad effects of stress may cause severe illness including diabetes and dementia (stress related disorders). However, despite of the progression of human knowledge, there is limitations in cure of the these disorders. In this study, we try to reduce the effects of stress on memory deficit, using the drug memantine. Our data suggested that memantine reduces the bad effects of stress on learning and memory, which could be used in some cases in other animals and human as well.

\section{Introduction}

tress is considered one of the major factors involved in brain disorders, including dementia and Alzheimer disease (Lupien Juster, Raymond, \& Marin, 2018; Lupien McEwen, Gunnar, \& Heim, 2009). A huge amount of data indicated that the stress mediators, including the glucocorticoid hormones, such as cortisol and corticosterone are involved in the brain compartments deformation, which results in the exacerbation of the functional deficits (McEwen, Nasca, \& Gray, 2016). In this regard, Vyas and colleagues have shown that severe stress reduces the dendritic arborization of the pyramidal neurons in the rat hippocampus (Vyas, Mitra, Rao, \& Chattarji, 2002). Also, it is indicated that economic stress is associated with the reduction of cognitive functions in humans, and this reduction is directly related to the hippocampus activity (Lynch, Kaplan, \& Shema, 1997). However, the inverse changes observed in the Basolateral Amygdala (BLA) indicated the trophic action of stress mediators in this region (Vyas, Jadhav, \& Chattarji, 2006). These changes are attributed to the different effects of glucocorticoids on the release of the Brain-Derived Neurotrophic Factor (BDNF) as the main trophic factor in these regions (Lakshminarasimhan \& Chattarji, 2012; Magarinos et al., 2011; Smith, Makino, Kvetnansky, \& Post, 1995).

The role of the hippocampus in learning and memory is well understood (Jarrard, 1993); this role is evidenced to be mediated, at least partially by the N-methyl-D-aspartate (NMDA) glutamate receptors of the hippocampus (Tsien, Huerta, \& Tonegawa, 1996). Also, the neurogen- esis in the hippocampus is critical for the normal function of learning and memory (Snyder, Hong, McDonald, \& Wojtowicz, 2005). Interestingly, both glutamate NMDA receptors and neurogenesis are affected by the stress hormones within the hippocampus (Babic et al., 2012; Popoli et al., 2012). Thus, it is not surprising that the inhibition of the NMDA receptors restrains the stress effects on the hippocampus morphological deformation (Babic, Ondrejcakova, Bakos, Racekova, \& Jezova 2012; Volbracht, Van Beek, Zhu, Blomgren, \& Leist, 2006).

Several studies have indicated that the NMDA receptors antagonists inhibit the stress-induced depression and anxiety in the animal models (Adamec, Burton, Shallow, \& Budgell, 1998; Garcia et al., 2008; Jessa, Nazar, \& Płaźnik,1995; Martínez et al., 2002). Also, the NMDA receptor antagonists may have anxiolytic and antidepressant effects in the human (Amidfar, Réus, Quevedo, \& Kim, 2018; Battista, Hierholzer, Khouzam, Barlow, \& O’Toole, 2007; Parsons, Danysz, \& Quack, 1999; Schwartz, Siddiqui, \& Raza, 2012). Some evidence suggests a functional interaction between the BLA and hippocampus. Zheng and colleagues have shown a functional dynamic interaction between the amygdala and hippocampus during the data processing in the human (Zheng et al., 2017). Moreover, the amygdala is the main brain compartment involved in the emotional memory processing (Adlolphs, 2003; McGaugh, 2004; Roozendaal, McEwen, \& Chattarji, 2009).

Considering these facts, it is not surprising that chronic stress can induce spatial learning and memory disturbances (Conrad, 2010). However, the literature did not specify whether the spatial learning and memory would 
change if the NMDA receptors of the BLA were blocked during the stress. This study aimed to clarify this issue using the electro-foot-shock as the stressor and memantine as the NMDA glutamate receptor blocker, while the animals were tested for their spatial learning and memory on the Barnes maze apparatus.

\section{Materials and Methods}

\subsection{Animals}

A total number of 111 male Wistar rats (Average weight $=200 \mathrm{~g}$; Pasture Institute, Tehran, Iran) were used throughout the study. The animals were kept in cages (4 animals/cage), also, the temperature $\left(22 \pm 2{ }^{\circ} \mathrm{C}\right)$ was maintained constant before and during the experiments. They have ad lib access to the standard rat chow and tap water, except during the experiments. Each animal in the experimental groups $(n=7)$ was used once in the experiments. All of the experiments were carried out according to the animal care guideline, Baqiyatallah Medical University Animal Ethics Committee.

\subsection{Drugs}

Ketamine hydrochloride (Alphasan, Holland), diazepam hydrochloride (Sigma, USA), and memantine hydrochloride (Tocris, England) were used. Drugs were dissolved in the sterile saline; the resulted solutions were used in a volume of $1 \mathrm{~mL}$ per each $\mathrm{kg}$ of the bodyweight for ketamine and diazepam, and $0.5 \mu \mathrm{L}$ per each rat for memantine.

\subsection{Surgical procedures}

Animals were anesthetized under ketamine $(70 \mathrm{mg} / \mathrm{kg})$ plus diazepam $(5 \mathrm{mg} / \mathrm{kg})$ for surgical procedures. For the BLA bilateral cannulation, two 23-gauge stainless steel guide cannulas were implanted bilaterally $0.5 \mathrm{~mm}$ above the injection site, according to the Paxinos and Watson atlas for the rat brain (Paxinos \& Watson, 2007). The coordinates of the BLA were as follows: $-3.3 \mathrm{~mm}$ (incisor bar), $-2.8 \mathrm{~mm}$ from the bregma, $\pm 5 \mathrm{~mm}$ lateral from the middle line, and $6.8 \mathrm{~mm}$ deep from the skull surface. The cannulas were secured using two stainless steel screws and the dental acrylic resin. Two dummy cannulas (stainless steel wire, $0.1 \mathrm{~mm}$ diameter) were inserted into the guide cannulas for possible obstruction. These dummy cannulas remained in the guide cannulas until the injections. The length of the dummy cannulas was matched with that of the guide cannulas. The animals were allowed seven days to recover from the side effects of the surgery and anesthesia.
For drug injections, each animal was restrained, and the dummy cannulas were removed, then, two 30-gauge injection cannulas whose tips were $500 \mu \mathrm{m}$ below the tip of the guide cannulas were inserted into the guide cannulas. The memantine solutions were slowly injected into the BLA in a total volume of $0.5 \mu \mathrm{L}$ per each rat $(0.25 \mu \mathrm{L}$ in each side) for 60 seconds. The injection cannulas were left in the guide cannulas for an additional 60 seconds to facilitate the drug diffusion.

\subsection{The electric foot-shock stress procedure}

The animals were placed in the communication box (Borje Sanat Co., Tehran, Iran); after 30 minutes, they experienced an electro-foot-shock $(40 \mathrm{mV}, 10 \mathrm{~Hz})$ for 100 seconds. Thirty minutes later, the animals were returned to their home cages. This procedure was repeated for seven consecutive days. On the eighth day, the animals were tested for their spatial learning and memory on the Barnes maze.

\subsection{Blood sampling}

On the seventh day of the stress sessions, the blood samples were taken from the retro-orbital sinus of the animals $(0.5 \mathrm{~mL}$ of blood in $0.5 \mathrm{~mL}$ of $1 \%$ sodium citrate), between 13:00 and 13:30. The samples were centrifuged in $2500 \times \mathrm{g}$ at $4^{\circ} \mathrm{C}$ for 5 minutes, then, the supernatant serum was collected for corticosterone detection. The corticosterone concentration was determined by the ELISA kit (Rat Corticosterone ELISA kit, EIA-4164, DRG Instruments GmbH, Germany) at $450 \mathrm{~nm}$.

\subsection{Spatial learning and memory testing}

The spatial learning and memory tests were performed using a Barnes maze positioned in a sound-attenuating experimental room, according to Maghami and colleagues with minor modifications (Maghami et al., 2018). The maze platform (dia=100 $\mathrm{cm}$ ) was made by opaque blue circular Plexiglas with 18 holes (dia=10 $\mathrm{cm})$ placed at the edge of the platform with equal spacing. The platform stood on a base with a height of 120 $\mathrm{cm}$ from the ground. Also, an escape box $(20 \times 20 \times 20$ $\mathrm{cm}$ ) made by black Plexiglas was attached to one of the holes and considered as a target hole. The target hole had the same position for every animal throughout the test. The black strips with different shapes were attached to the walls of the experimental room, for spatial cues. Besides, the experimenter was hiding behind a curtain during the experiments. 
The animals' activity on the maze was monitored and recorded using a CCTV camera located $90 \mathrm{~cm}$ above the maze platform. This device recorded the animals' activity and the software developed by Borje Sanat Co., Tehran, Iran, analyzed the animals' movement on the maze. The software provided all the study parameters, including time, distance traveled by the animal, the number of errors (the animal movements at the edge of a hole other than the target hole), and the strategy the animal took to reach the target hole (rats used three types of strategies, namely direct, serial, and random to reach the target hole). The direct strategy means that the animal moves directly from the center of the maze to the escape box. The serial strategy means that the animal moves serially between the holes until it reaches the escape box. The random strategy means that the animal moves randomly in the center of the maze until it reaches the escape box.

Each animal experienced four trials per day. For this purpose, each animal was brought to the test room, 60 minutes before the learning trial. Next, it was put in the center of the maze under a black bucket, while the lights were off. Then, the lights were turned on, the bucket was removed, and the animal was allowed to find the target hole in 90 seconds (cut-off time). If the animal did not find the target hole in this time, it would be guided manually to the target hole by the experimenter. To habituate the animals to the maze environment, one day before starting the learning trials, the animals were put in the escape box for two minutes, then, placed directly in the target hole and allowed to enter and stay in the escape box, beneath the hole, for two minutes.

When the animals entered the target hole, they were allowed to stay there for two minutes, then, were returned to its cage for 15 minutes. After each trial session, the maze and the target hole were cleaned using ethanol $70 \%$. This procedure was repeated for five consecutive days. On the sixth day, each animal was placed on the maze while the target hole was covered by a dark plate. The animal was allowed to freely move on the maze for 90 seconds. The time the animal spent on the dark plate was recorded as an indicator of the spatial memory index.

\subsection{Histology}

After the completion of the experiment, the animals were deeply anesthetized with high doses of the ketamine hydrochloride, then, a methylene blue $4 \%$ solution was injected into the guide cannula at a volume of $0.25 \mu \mathrm{L}$ per each side. The animals' brains were removed surgically, after the infusion of the transcardiac cold saline $\left(4^{\circ} \mathrm{C}\right)$ and formalin $4 \%$. The brains were dissected, and the site of the injection was determined by a professional anatomist. Only the results of those animals in which the injection site was correctly performed were considered for further statistical analyses (Figure 1).

\subsection{Statistical analysis}

The obtained data were shown as Mean \pm SEM. Also, the area under the curve was calculated for the spent time and traveled distance for reaching the target hole. Using the stress and memantine as the analyzing factors, the two-way mixed ANOVA followed by the Tukey post hoc test was conducted to analyze the data. In all cases, the $P$ value of less than 0.05 was considered a statistically significant result.

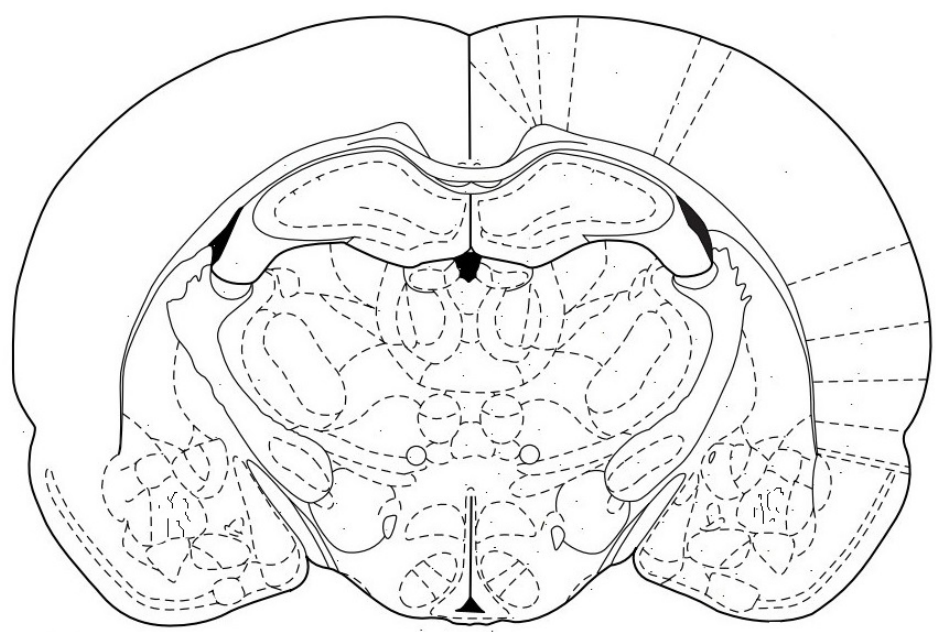

Figure 1. Injection site of memantine in the basolateral amygdala 


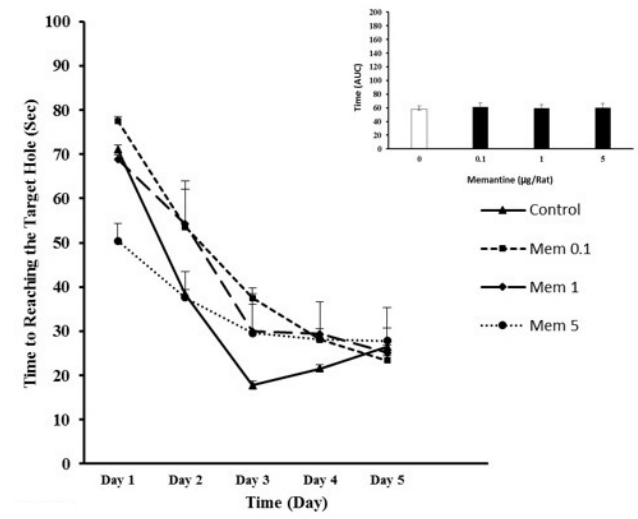

C

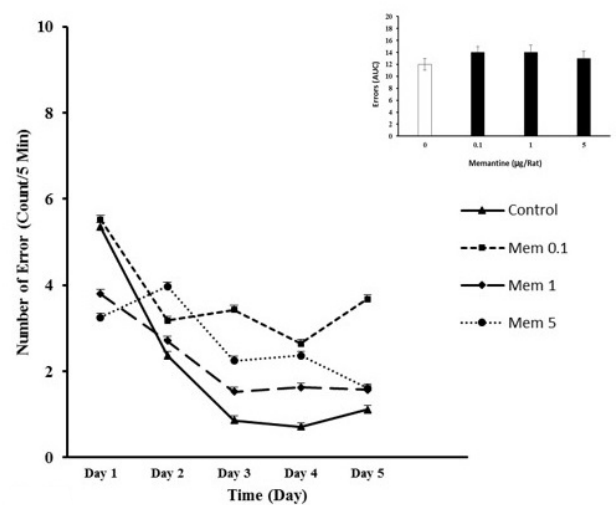

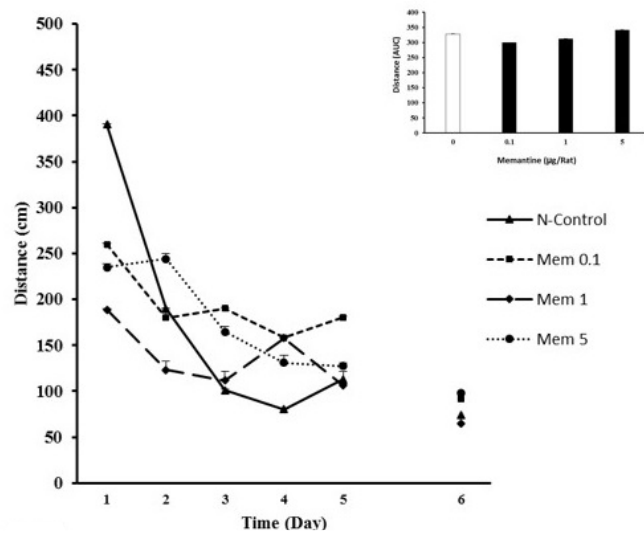

D

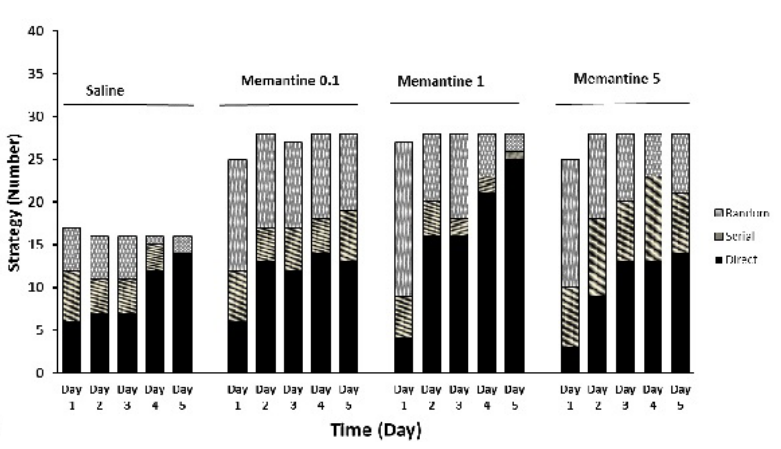

NEUR SCIENCE

Figure 2. Effect of the intra-BLA memantine administration on the parameters of spatial learning and memory in unstressed rats

A: Figures show the elapsed time; B: Distance traveled to reach the target hole; C: The number of errors; and D: The strategy taken by the animals to reach the target hole in unstressed rats. The rats received different doses of memantine $(0.1,1$, and $5 \mu \mathrm{g} / \mathrm{rat})$ or saline $(0.25 \mu \mathrm{L} /$ side) for seven consecutive days, then, they were evaluated for spatial learning and memory on the Barnes maze $(n=6-8)$.

\section{Results}

3.1. Evaluation of the effect of memantine administration on spatial learning and memory in the unstressed rats

Four groups of the animals $(\mathrm{n}=6-8)$ were cannulated bilaterally in the BLA. After seven days, they received intraBLA memantine $(0.1,1$, and $5 \mu \mathrm{g} / \mathrm{rat})$ or saline $(0.5 \mu \mathrm{L} / \mathrm{rat})$, once a day for seven consecutive days. On the eighth day, the animals were tested for spatial learning and memory on the Barnes maze. Our data indicated that the intra-BLA memantine did not change the spent time and distance traveled to the target hole, the number of errors, and the strategy (Figure 2, A-D).
3.2. The effects of the intra-BLA memantine administration on spatial learning and memory in the stressed rats

Four groups of the animals were cannulated. Seven days later, they experienced electro-foot-shock once a day for seven consecutive days. Memantine $(0.1,1$, and $5 \mu \mathrm{g} / \mathrm{rat})$ or saline $(0.5 \mu \mathrm{L} / \mathrm{rat})$ was injected into the BLA, five minutes before each stress session. The obtained data indicated that stress increased the spent time and distance traveled by the animals for reaching the target hole. Also, stress increased the number of errors. Interestingly, stress changed the animals' strategy to random type. However, memantine $(0.1,1$, and 5 $\mu \mathrm{g} / \mathrm{rat}$ ) reduced all the stress effects (Figure 3, A-D).

\subsection{Evaluation of the plasma corticosterone level}

On the seventh day of the experiment, the blood samples were taken from the retro-orbital sinus of the study animals. The data showed that stress significantly increased the plas- 


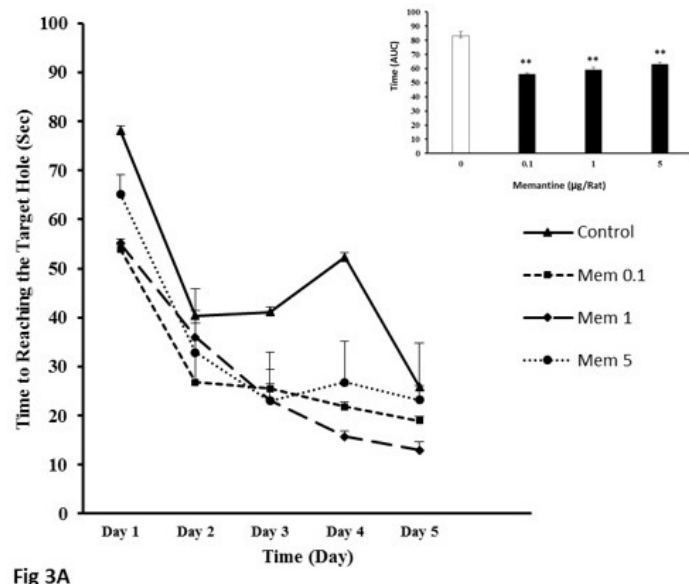

Fig 3 A

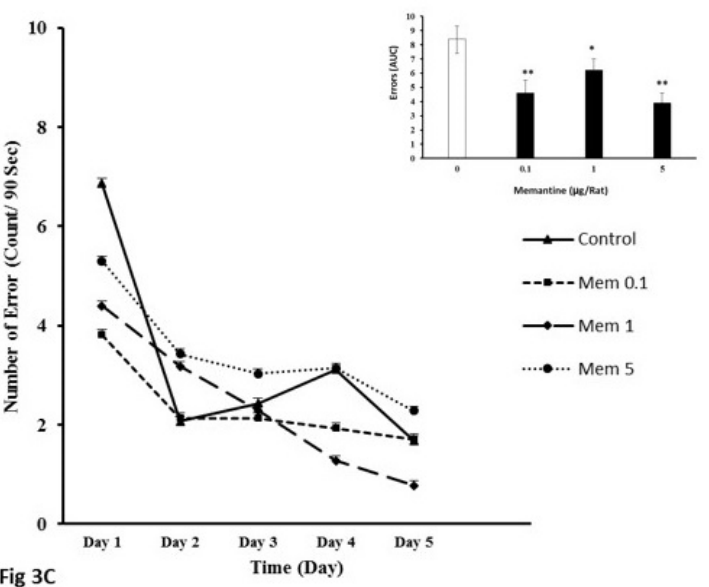

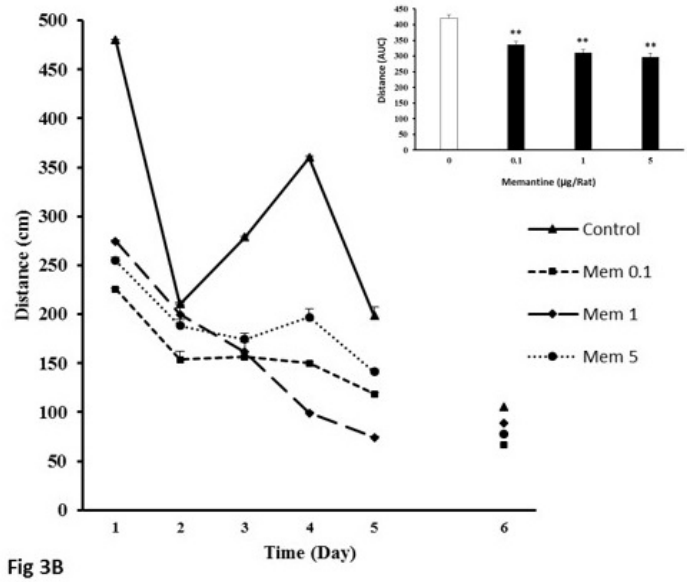

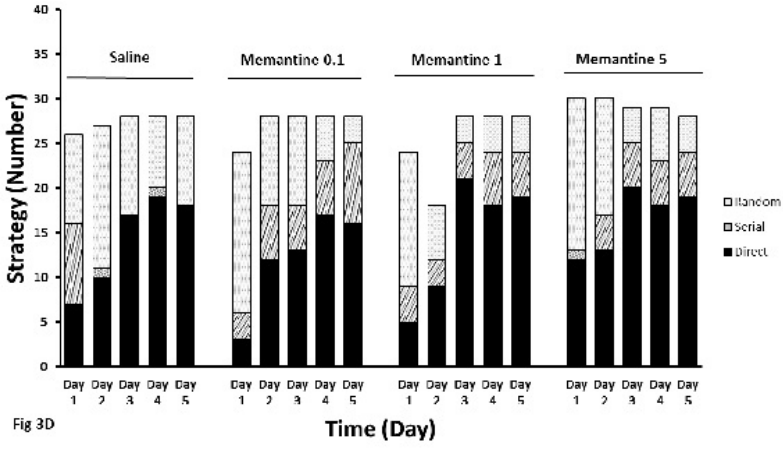

NEUR SCIENCE

Figure 3. Effect of the intra-BLA memantine administration on the parameters of spatial learning and memory in stressed rats

A: Figures show the time to reach the target hole; B: Distance traveled for reaching the target hole; C: The number of errors; and D: The strategy taken by the animals to reach the target hole. The rats received the different doses of memantine $(0.1,1, \mathrm{and} 5 \mu \mathrm{g} / \mathrm{rat})$ or saline $(0.25 \mu \mathrm{L} /$ side), five minutes before the induction of stress for seven consecutive days, then, they were evaluated for spatial learning and memory on the Barnes maze $(n=6-8)$.

$* \mathrm{P}<0.05$;

$* * \mathrm{P}<0.01$.

ma corticosterone levels $(\mathrm{P}<0.001$; Figure 4). However, memantine administration reduced the stress effects.

\section{Discussion}

This study aimed to further clarify the role of glutamate NMDA receptors of the BLA on the chronic stressinduced disturbance in spatial learning and memory. Our data indicated that memantine as an antagonist of the NMDA receptors did not change the learning and memory function when administered in the BLA of unstressed rats. However, when the drug administered before the stress sessions, it reduced the stress effects on learning and memory destruction, indicating the crucial role of the NMDA receptors within the BLA on this issue.

It is generally accepted that spatial learning and memory is one of the important functions of the dorsal hippocampus (Eichenbaum, 2004; McEwen \& Sapolsky, 1995), and can be influenced by environmental factors, including stressful events (de Kloet, Karst, \& Joëls, 2008; Makhathini, Abboussi, Stein, Mabandla, \& Daniels, 2017). Our data indicated that chronic (seven days) electro-foot-shock stress applied unpredictably impaired the spatial learning and memory, as evaluated in the Barnes maze apparatus. Accordant with previ- 


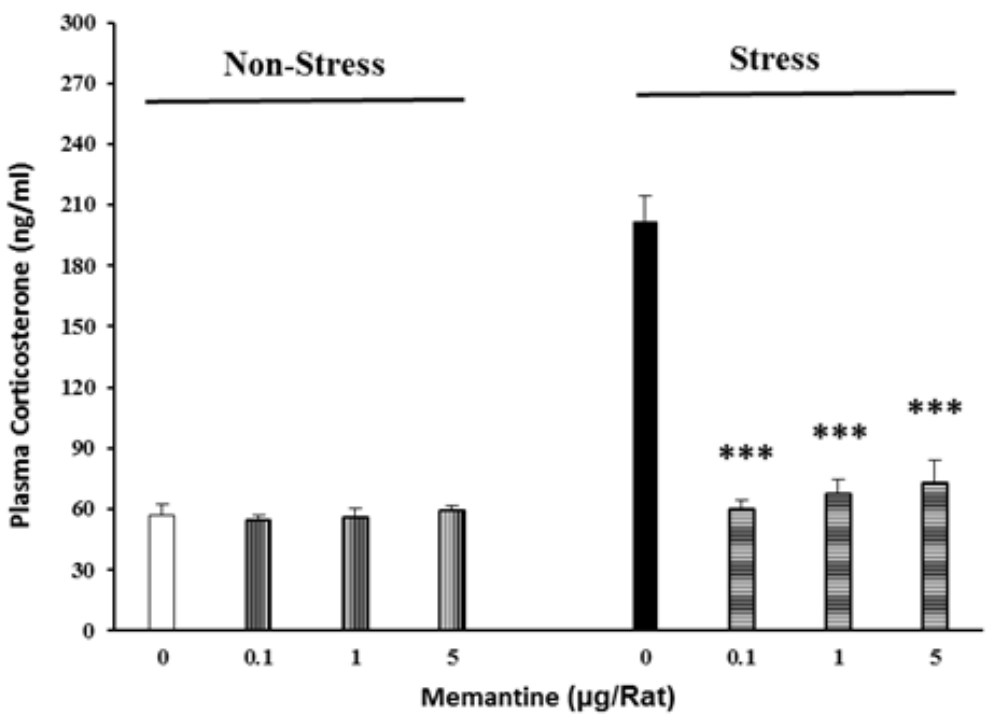

Figure 4. Effect of the chronic electro-foot-shock stress on plasma corticosterone level

ous studies (Asalgoo, Pirzad Jahromi, Hatef, \& Sahraei, 2018; Gawel, Gibula, Marszalek-Grabska, Filarowska, \& Kotlinska, 2019), our results showed that all factors, including the time to reach the target hole, the distance traveled for target hole achievement, and the number of errors increased in the stressed animals. Also, the strategy used by the animals for reaching the target hole was changed to the random type. The manner of these intense changes in memory performance indicated a significant impairment in spatial learning and memory.

Several studies have indicated that impairment in spatial learning and memory will occur in the rodents, after chronic corticosterone administration or chronic stress experience (Conrad, 2010; McLay, Freeman, \& Zadina, 1998; Sandi, 1998; Sandi, 2004; Sandi, 2011; Hosseinmardi, Shiravi, Meftahi, \& Afarinesh, 2019). The authors have postulated that glucocorticoids released during stressful events accelerate morphological deformation and functional retardation in the hippocampal pyramidal cells. These deformations include a massive reduction in the dendritic tree and cell body reduction because of the hormones. They act on the receptors located both in the cell cytoplasm and the cell membrane surface of the hippocampal pyramidal neurons (McEwen et al., 2016; McEwen, 1999; McEwen, 2000; Popoli, Yan, McEwen, \& Sanacora, 2012; Hashtjin et al., 2018).

These morphological changes in the hippocampus result in the decline of cognitive function, including a reduction in spatial learning and memory (Jarrard, 1983; Jarrard, 1978; Kim \& Diamond, 2002; Landfield, Waymire, \& Lynch, 1978; McEwen \& Sapolsky, 1995;
Aliabadi, Sahraei, Bahari, \& Meftahi, 2018). A similar mechanism(s) may be involved in the results of the present study. We introduced a shorter stress-induction paradigm using the electro-foot-shock, compared with previous studies that used intense restrain paradigm in this issue (i.e., $6 \mathrm{~h} / \mathrm{d}$ restrain stress for 21 days) (Lakshminarasimhan \& Chattarji, 2012).

Memantine is a low affinity and noncompetitive antagonist of the NMDA glutamate receptor (Song et al., 2018), and has positive effects in cognitive diseases, including Alzheimer disease (Reisberg et al., 2003; Tariot et al., 2004; Volbracht et al., 2006). Our data indicated that memantine reduces the adverse effects of stress on spatial learning and memory when injected into the BLA of the rats. The functional relationship between the BLA and hippocampus is well understood. Using the fearful face paradigm and the recording of gamma waves, Zheng and colleagues have shown a similar pattern of electrical activity in the amygdala and hippocampus during information processing, in the human (Zheng et al., 2017).

Besides, Roosendaal et al., have shown that the BLA is essential for memory consolidation in the hippocampus (Roozendaal et al., 2003; Roozendaal et al., 2009). Interestingly, the efficacy of glucocorticoids in reducing spatial memory depends on the activity of the BLA (Roozendaal, Griffith, Buranday, Dominique, \& McGaugh, 2003; Yang et al., 2007; Hosseinmardi et al., 2019). In other words, the memory enhancement function of the glucocorticoid in the dorsal hippocampus requires the BLA to be functional (Roozendaal \& McGaugh, 1997). 
The glucocorticoids activate glutamate receptors and neurotransmission this affecting learning and memory (Sandi, 2011). Considering these facts, it is expected that the intra-BLA memantine administration before each stress session inhibited the stress-induced spatial learning and memory impairment. Other studies have focused on the effects of the different antagonists of NMDA glutamate receptors, including MK-801 (de Lima, Laranja, Bromberg, Roesler, \& Schröder, 2005) and ketamine (Newcomer et al., 1999). Our results indicated that memantine is effective for the reduction of stress hormones' impacts on the BLA, and improves spatial learning and memory.

The present study indicated that stress changed the strategy adopted by the animals for reaching the target hole. It is well known that strategy is a complex phenomenon that is needed to be pluralized in the prefrontal cortex (Ragozzino, Wilcox, Raso, \& Kesner,1999; Rich \& Shapiro, 2009). Moreover, glucocorticoids can impair memory by direct action on the prefrontal cortex (Barsegyan, Mackenzie, Kurose, McGaugh, \& Roozendaal, 2010). Also, the evidence indicates that glucocorticoid function in memory relies on the interaction of the BLA and prefrontal cortex (Roozendaal et al., 2009). Altogether, these data indicate that stress interacts with the prefrontal cortex both directly and indirectly by the mediation of the BLA to change the strategy type. However, memantine inhibits the stress effect on strategy; it is an important finding because severe social cognitive phenomena may be related to this function of the prefrontal cortex (Adlolphs, 2003).

Eventually, chronic electro-foot-shock stress can impair spatial learning and memory, as revealed by the Barnes maze paradigm. However, the pre-microinjection of memantine into the BLA altered these impairments. Stress changes the strategy used by the rats to achieve the target hole; memantine also inhibited this effect. Considering the effectiveness of memantine on the inhibition of stress effect on spatial learning and memory, it is recommended to test the drug in other paradigms of spatial learning and memory testing.

\section{Ethical Considerations}

\section{Compliance with ethical guidelines}

All the experiments were conducted according to the animal care guidelines of the Animal Ethics Committee of Baqiyatallah Medical University (Ethical Code: 3225 : November 2016).

\section{Funding}

This research did not receive any specific grant from funding agencies in the public, commercial, or non-profit sectors.

\section{Authors' contributions}

Investigation, and writing-original draft: Ahmad Rafia, Hedayat Sahraei; Conceptualization, formal analysis: Ahmad Rafia, Hedayat Sahraei; Data curation, methodology: All authors; Writing, reviewing, and editing: Hedayat Sahraei, Shahrbanoo Oryan, Akram Eidi; Visualization, supervision, project administration: Hedayat Sahraei.

\section{Conflict of interest}

The authors declared no conflict of interest.

\section{References}

Adamec, R. E., Burton, P., Shallow, T., \& Budgell, J. (1998). Unilateral block of NMDA receptors in the amygdala prevents predator stress-induced lasting increases in anxiety-like behavior and unconditioned startle-effective hemisphere depends on the behavior. Physiology \& Behavior, 65(4-5), 739-51. [DOI:10.1016/ S0031-9384(98)00225-X

Adlolphs, R. (2003). Cognitive neuroscience of human social behavior. Nature Reviews Neuroscience, 4(3):165-78. [DOI:10.1038/ nrn1056] [PMID]

Aliabadi, N., Sahraei, H., Bahari, Z., \& Meftahi, G. (2018). Effect of prenatal immobilization stress on spatial memory, anxiety-like behavior and brain BDNF concentration in the F1 generation male mice. Scientific Journal of Kurdistan University of Medical Sciences, 23(3), 73-84. https://www.cabdirect.org/cabdirect/abstract/20183355399

Amidfar, M., Réus, G. Z., Quevedo, J., \& Kim, Y. K. (2018). The role of memantine in the treatment of major depressive disorder: Clinical efficacy and mechanisms of action. European Journal of Pharmacology, 827, 103-11. [DOI:10.1016/j.ejphar.2018.03.023] [PMID]

Asalgoo, S., Pirzad Jahromi, G., Hatef, B., \& Sahraei, H. (2018). The effect of saffron aqueous extract and crocin on PTSD rat models: The focus on learning and spatial memory. Journal of Advances in Medical and Biomedical Research, 26(119), 34-42. [DOI:10.30699/ jambs.26.119.34]

Babic, S., Ondrejcakova, M., Bakos, J., Racekova, E., \& Jezova, D. (2012). Cell proliferation in the hippocampus and in the heart is modified by exposure to repeated stress and treatment with memantine. Journal of Psychiatric Research, 46(4), 526-32. [DOI:10.1016/j.jpsychires.2012.01.002] [PMID]

Barsegyan, A., Mackenzie, S. M., Kurose, B. D., McGaugh, J. L., \& Roozendaal, B. (2010). Glucocorticoids in the prefrontal cortex 
enhance memory consolidation and impair working memory by a common neural mechanism. Proceedings of the National Academy of Sciences of the United States of America, 107(38), 1665560. [DOI:10.1073/pnas.1011975107] [PMID] [PMCID]

Battista, M. A., Hierholzer, R., Khouzam, H. R., Barlow, A., \& O'Toole, S. (2007). Pilot trial of memantine in the treatment of posttraumatic stress disorder. Psychiatry, 70(2), 167-74. [DOI:10.1521/psyc.2007.70.2.167] [PMID]

Conrad, C. (2010). A critical review of chronic stress effects on spatial learning and memory. Progress in Neuro-Psychopharmacology \& Biological Psychiatry, 34(5), 742-55. [DOI:10.1016/j.pnpbp.2009.11.003] [PMID]

de Kloet, E. R., Karst, H., \& Joëls, M. (2008). Corticosteroid hormones in the central stress response: Quick-and-slow. Frontiers in Neuroendocrinology, 29(2), 268-72. [DOI:10.1016/j.yfrne.2007.10.002] [PMID]

de Lima, M. N. M., Laranja, D. C., Bromberg, E., Roesler, R., \& Schröder, N. (2005). Pre-or post-training administration of the NMDA receptor blocker MK-801 impairs object recognition memory in rats. Behavioural Brain Research, 156(1), 139-43. [DOI:10.1016/j.bbr.2004.05.016] [PMID]

Eichenbaum, H. (2004). Hippocampus: Cognitive processes and neural representations that underlie declarative memory. Neuron, 44(1), 109-20. [DOI:10.1016/j.neuron.2004.08.028] [PMID]

Garcia, L. S., Comim, C. M., Valvassori, S. S., Réus, G. Z., Barbosa, L. M., \& Andreazza, A. C., et al. (2008). Acute administration of ketamine induces antidepressant-like effects in the forced swimming test and increases BDNF levels in the rat hippocampus. Progress in Neuro-Psychopharmacology \& Biological Psychiatry, 32(1), 140-4. [DOI:10.1016/j.pnpbp.2007.07.027] [PMID]

Gawel, K., Gibula, E., Marszalek-Grabska, M., Filarowska, J., \& Kotlinska, J. H. (2019). Assessment of spatial learning and memory in the Barnes maze task in rodents-methodological consideration. Naunyn-Schmiedeberg's Archives of Pharmacology, 392(1), 1-18. [DOI:10.1007/s00210-018-1589-y] [PMID] [PMCID]

Hashtjini, M. M., Jahromi, G. P., Sadr, S. S., Meftahi, G. H., Hatef, B., \& Javidnazar, D. (2018). Deep brain stimulation in a rat model of post-traumatic stress disorder modifies forebrain neuronal activity and serum corticosterone. IIranian Journal of Basic Medical Sciences, 21(4), 370-5. [DOI:10.22038/IJBMS.2018.27482.6705]

Hosseinmardi, L., Shiravi, A., Meftahi, G. H., \& Afarinesh, M. R. (2019). Inactivation of $\beta 1$-adrenergic receptor in the basolateral amygdala nucleus attenuated anxiety-like behaviour in response to foot-shock stress in the male rat. Physiology and Pharmacology, 23(2), 101-14. http://ppj.phypha.ir/article-1-1421-en.pdf

Jarrard, L. E. (1993). On the role of the hippocampus in learning and memory in the rat. Behavioral and Neural Biology, 60(1), 9-26. [DOI:10.1016/0163-1047(93)90664-4]

Jarrard, L. E. (1983). Selective hippocampal lesions and behavior: Effects of kainic acid lesions on performance of place and cue tasks. Behavioral neuroscience, 97(6), 873-89. [DOI:10.1037/07357044.97.6.873] [PMID]

Jarrard, L. E. (1978). Selective hippocampal lesions: differential effects on performance by rats of a spatial task with preoperative versus postoperative training. Journal of Comparative and Physiological Psychology, 92(6), 1119. [DOI:10.1037/h0077516] [PMID]
Jessa, M., Nazar, M., \& Płaźnik, A. (1995). Anxiolytic-like action of intra-hippocampally administered NMDA antagonists in rats. Polish Journal of Pharmacology, 47(1), 81-84. [PMID]

Kim, J. J., \& Diamond, D. M. (2002). The stressed hippocampus, synaptic plasticity and lost memories. Nature Reviews Neuroscience, 3(6), 453. [DOI:10.1038/nrn849] [PMID]

Lakshminarasimhan, H., \& Chattarji, S. (2012). Stress leads to contrasting effects on the levels of brain derived neurotrophic factor in the hippocampus and amygdala. PLoS One, 7(1), e30481. [DOI:10.1371/journal.pone.0030481] [PMID] [PMCID]

Landfield, P. W., Waymire, J., \& Lynch, G. J. S. (1978). Hippocampal aging and adrenocorticoids: Quantitative correlations. Science, 202(4372), 1098-102. [DOI:10.1126/science.715460] [PMID]

Lupien, S. J., Juster, R. P., Raymond, C., \& Marin, M. F. (2018). The effects of chronic stress on the human brain: from neurotoxicity, to vulnerability, to opportunity. Frontiers in Neuroendocrinology, 49, 91-105. [DOI:10.1016/j.yfrne.2018.02.001] [PMID]

Lupien, S. J., McEwen, B. S., Gunnar, M. R., \& Heim, C. (2009). Effects of stress throughout the lifespan on the brain, behaviour and cognition. Nature Reviews Neuroscience, 10(6), 434. [DOI:10.1038/ nrn2639] [PMID]

Lynch, J. W., Kaplan, G. A., \& Shema, S. J. (1997). Cumulative impact of sustained economic hardship on physical, cognitive, psychological, and social functioning. The New England Journal of Medicine, 337(26), 1889-95. [DOI:10.1056/NEJM199712253372606] [PMID]

Magarinos, A., Li, C., Toth, J. G., Bath, K., Jing, D., \& Lee, F., et al. (2011). Effect of brain-derived neurotrophic factor haploinsufficiency on stress-induced remodeling of hippocampal neurons. Hippocampus, 21(3), 253-64. [DOI:10.1002/hipo.20744] [PMID] [PMCID]

Maghami, S., Zardooz, H., Khodagholi, F., Binayi, F., Saber, R. R., \& Hedayati, M., et al. (2018). Maternal separation blunted spatial memory formation independent of peripheral and hippocampal insulin content in young adult male rats. PloS One, 13(10), e0204731. [DOI:10.1371/journal.pone.0204731] [PMID] [PMCID]

Makhathini, K. B., Abboussi, O., Stein, D. J., Mabandla, M. V., \& Daniels, W. M. (2017). Repetitive stress leads to impaired cognitive function that is associated with DNA hypomethylation reduced $\mathrm{BDNF}$ and a dysregulated HPA axis. International Journal of Developmental Neuroscience, 60, 63-9. [DOI:10.1016/j. ijdevneu.2017.04.004] [PMID]

Martínez, G., Ropero, C., Funes, A., Flores, E., Blotta, C., \& Landa, A I., et al. (2002). Effects of selective NMDA and non-NMDA blockade in the nucleus accumbens on the plus-maze test. Physiology \& Behavior, 76(2), 219-24. [DOI:10.1016/S0031-9384(02)00704-7]

McEwen, B. S., Nasca, C., \& Gray, J. D. (2016). Stress effects on neuronal structure: hippocampus, amygdala, and prefrontal cortex. Neuropsychopharmacology, 41(1), 3-23. [DOI:10.1038/ npp.2015.171] [PMID] [PMCID]

McEwen, B. S., \& Sapolsky, R. M. (1995). Stress and cognitive function. Current Opinion in Neurobiology, 5(2), 205-16 [DOI:10.1016/0959-4388(95)80028-X]

McEwen, B. S. (1999). Stress and hippocampal plasticity. Annual Review of Neuroscience, 22(1), 105-22. [DOI:10.1146/annurev.neuro.22.1.105] [PMID] 
McEwen, B. S. (2000). Effects of adverse experiences for brain structure and function. Biological Psychiatry, 48(8), 721-31. [DOI:10.1016/S0006-3223(00)00964-1]

McGaugh, J. L. (2004). The amygdala modulates the consolidation of memories of emotionally arousing experiences. Annual Review of Neuroscience, 27, 1-28. [DOI:10.1146/annurev.neuro.27.070203.144157] [PMID]

McLay, R. N., Freeman, S. M., Zadina, J. E. (1998). Chronic corticosterone impairs memory performance in the Barnes maze. Physiology \& Behavior, 63(5), 933-7. [DOI:10.1016/S0031-9384(97)005295]

Newcomer, J. W., Farber, N. B., Jevtovic-Todorovic, V., Selke, G., Melson, A. K., \& Hershey, T., et al. (1999). Ketamine-induced NMDA receptor hypofunction as a model of memory impairment and psychosis. Neuropsychopharmacol, 20(2), 106-18. [DOI:10.1016/S0893-133X(98)00067-0]

Parsons, C., Danysz, W., \& Quack, G. (1999). Memantine is a clinically well tolerated N-methyl-D-aspartate (NMDA) receptor antagonist: A review of preclinical data. Neuropharmacology, 38(6), 735-67. [DOI:10.1016/S0028-3908(99)00019-2]

Paxinos, G., \& Watson, C. (2007). The rat brain in stereotaxic coordinates, $6^{\text {th }}$ ed. Philadelphia: Elsevier.

Popoli, M., Yan, Z., McEwen, B. S., \& Sanacora, G. (2012). The stressed synapse: the impact of stress and glucocorticoids on glutamate transmission.Nature Reviews Neuroscience, 13(1), 22. [DOI:10.1038/nrn3138] [PMID] [PMCID]

Ragozzino, M. E., Wilcox, C., Raso, M., \& Kesner, R. P. (1999). Involvement of rodent prefrontal cortex subregions in strategy switching. Behavioral neuroscience, 113(1), 32. [DOI:10.1037/07357044.113.1.32] [PMID]

Reisberg, B., Doody, R., Stöffler, A., Schmitt, F., Ferris, S., \& Möbius, H. J. (2003). Memantine in moderate-to-severe Alzheimer's disease. The New England Journal of Medicine, 348(14), 1333-41. [DOI:10.1056/NEJMoa013128] [PMID]

Rich, E. L., \& Shapiro, M. J. (2009). Rat prefrontal cortical neurons selectively code strategy switches. The Journal of Neuroscience, 29(22), 7208-19. [DOI:10.1523/JNEUROSCI.6068-08.2009] [PMID] [PMCID]

Roozendaal, B., Griffith, Q. K., Buranday, J., Dominique, J. F., \& McGaugh, J. L. (2003). The hippocampus mediates glucocorticoid-induced impairment of spatial memory retrieval: Dependence on the basolateral amygdala. Proceedings of the National Academy of Sciences of the United States of America, 100(3), 1328-33. [DOI:10.1073/pnas.0337480100] [PMID] [PMCID]

Roozendaal, B., McEwen, B. S., \& Chattarji, S. (2009). Stress, memory and the amygdala. Nature Reviews Neuroscience, 10(6), 423-33. [DOI:10.1038/nrn2651] [PMID]

Roozendaal, B., \& McGaugh, J. L. (1997). Basolateral amygdala lesions block the memory-enhancing effect of glucocorticoid administration in the dorsal hippocampus of rats. European Journal of Neuroscience, 9(1), 76-83. [DOI:10.1111/j.1460-9568.1997. tb01355.x] [PMID]

Roozendaal, B., McReynolds, J. R., Van der Zee, E. A., Lee, S., McGaugh, J. L., \& McIntyre, C. K. (2009). Glucocorticoid effects on memory consolidation depend on functional interactions between the medial prefrontal cortex and basolateral amygdala. The Journal of Neuroscience, 29(45), 14299-308. [DOI:10.1523/ JNEUROSCI.3626-09.2009] [PMID] [PMCID]
Sandi, C. (1998). The role and mechanisms of action of glucocorticoid involvement in memory storage. Neural Plasticity, 6(3), 4152. [DOI:10.1155/NP.1998.41] [PMID] [PMCID]

Sandi, C. (2004). Stress, cognitive impairment and cell adhesion molecules. Nature Reviews Neuroscience, 5(12), 917-30. [DOI:10.1038/ nrn1555] [PMID]

Sandi, C. (2011). Glucocorticoids act on glutamatergic pathways to affect memory processes. Trends in Neurosciences, 34(4), 165-76. [DOI:10.1016/j.tins.2011.01.006] [PMID]

Schwartz, T. L., Siddiqui, U. A., \& Raza, S. (2012). Memantine as an augmentation therapy for anxiety disorders. Case Reports in Psychiatry, 2012, 749796. [DOI:10.1155/2012/749796] [PMID] [PMCID]

Smith, M. A., Makino, S., Kvetnansky, R., \& Post, R. M. (1995). Stress and glucocorticoids affect the expression of brain-derived neurotrophic factor and neurotrophin-3 mRNAs in the hippocampus. The Journal of Neuroscience, 15(3), 1768-77. [DOI:10.1523/JNEUROSCI.15-03-01768.1995] [PMID] [PMCID]

Snyder, J., Hong, N., McDonald, R., \& Wojtowicz, J. (2005). A role for adult neurogenesis in spatial long-term memory. The Journal of Neuroscience, 130(4), 843-52. [DOI:10.1016/j.neuroscience.2004.10.009] [PMID]

Song, X., Jensen, M. Ø., Jogini, V., Stein, R. A., Lee, C.-H., \& Mchaourab, H. S., et al. (2018). Mechanism of NMDA receptor channel block by MK-801 and memantine. Nature, 556(7702), 51519. [DOI:10.1038/s41586-018-0039-9] [PMID] [PMCID]

Tariot, P. N., Farlow, M. R., Grossberg, G. T., Graham, S. M., McDonald, S., \& Gergel, I., et al. (2004). Memantine treatment in patients with moderate to severe Alzheimer disease already receiving donepezil: A randomized controlled trial. JAMA, 291(3) 317-24. [DOI:10.1001/jama.291.3.317] [PMID]

Tsien, J. Z., Huerta, P. T., \& Tonegawa, S. (1996). The essential role of hippocampal CA1 NMDA receptor-dependent synaptic plasticity in spatial memory. Cell, 87(7), 1327-38. [DOI:10.1016/S0092 8674(00)81827-9]

Volbracht, C., Van Beek, J., Zhu, C., Blomgren, K., \& Leist, M. (2006). Neuroprotective properties of memantine in different in vitro and in vivo models of excitotoxicity. European Journal of Neuroscience, 23(10), 2611-22. [DOI:10.1111/j.1460-9568.2006.04787.x] [PMID]

Vyas, A., Jadhav, S., \& Chattarii, S. (2006). Prolonged behaviora stress enhances synaptic connectivity in the basolateral amygdala. The Journal of Neuroscience, 143(2), 387-93. [DOI:10.1016/j. neuroscience.2006.08.003] [PMID]

Vyas, A., Mitra, R., Rao, B. S., \& Chattarii, S. (2002). Chronic stress induces contrasting patterns of dendritic remodeling in hippocampal and amygdaloid neurons. The Journal of Neuroscience, 22(15), 6810-8. [DOI:10.1523/JNEUROSCI.22-15-06810.2002] [PMID] [PMCID]

Yang, Y. L., Chao, P. K., Ro, L. S., Wo, Y. Y. P., \& Lu, K. T. (2007) Glutamate NMDA receptors within the amygdala participate in the modulatory effect of glucocorticoids on extinction of conditioned fear in rats. Neuropsychopharmacology, 32(5), 1042-51. [DOI:10.1038/sj.npp.1301215] [PMID]

Zheng, J., Anderson, K. L., Leal, S. L., Shestyuk, A., Gulsen, G., \& Mnatsakanyan, L., et al. (2017). Amygdala-hippocampal dynamics during salient information processing. Nat commun 8, 14413. [DOI:10.1038/ncomms14413] [PMID] [PMCID] 